\title{
Aplicación de la metodología clase inversa en la enseñanza de máquinas eléctricas avanzadas
}

\author{
Paula Bastida-Molina $^{\mathrm{a}^{*}}$, Carlos Vargas-Salgado $^{\mathrm{b}}$ Lina Montuori $^{\mathrm{c}}$ y Manuel Alcázar-Ortega ${ }^{\mathrm{d}}$ \\ ${ }^{a}$ Instituto Universitario de Investigación de Ingeniería Energética, Camino de Vera, s/n, Edificio 8E, Universitat \\ Politècnica de València paubasmo@etsid.upv.es, ${ }^{b}$ Departamento de Ingeniería Eléctrica, Camino de Vera, s/n, \\ Edificio 5E, Universitat Politècnica de València, carvarsa@upvnet.upv.es, ${ }^{c}$ Departamento de Termodinámica \\ Aplicada, Camino de Vera, s/n, Edificio 5J, 2a planta, Universitat Politècnica de València, lmontuori@upvnet.upv.es \\ $\mathrm{y}{ }^{\mathrm{d}}$ Departamento de Ingeniería Eléctrica, Camino de Vera, s/n, Edificio 5E, Universitat Politècnica de València, \\ malcazar@iie.upv.es
}

\begin{abstract}
University education is undergoing a dynamic process of change, from a traditional learning system to a more flexible one. Is in this context where the flipped teaching methodology arises, which includes two different phases: conceptual and collaborative. During the conceptual phase, developed outside the classroom, the student prepares him/herself for the lesson using the resources previously elaborated by the professor. During the collaborative phase, the student applies and reinforces the knowledge acquired during the conceptual phase in a cooperative way and during the class time. Hence, the professor simply guides the students. In this paper, we analyze the application of the flipped teaching methodology to the $1^{\text {st }}$ laboratory lesson of the course Advanced Electrical Machines, from the Bachelor Degree in Electrical Engineering (Polytechnic University of Valencia). In it, students should analyze six real electrical machines, which have been previously theoretically explained. Results from the qualifying test show the high academic performance of the lesson applying this methodology. Particularly, 75\% of the students passed the exam and 23\% of them did it with outstanding grade. Moreover, the survey's answers demonstrate the direct relationship between the development degree of the previous conceptual phase and the academic performance of the lesson.
\end{abstract}

Keywords: flipped teaching, flexible learning, independent learning, collaborative work, active students, Bloom's taxonomy.

\section{Resumen}

La educación universitaria está experimentando un proceso de cambio dinámico, desde un sistema de enseñanza tradicional hacia otro más flexible. En este contexto surge la metodología clase inversa, la cual comprende dos fases: conceptual y colaborativa. En la fase conceptual, desarrollada fuera del aula, el estudiante se prepara para la clase a partir de los recursos que el docente elabora previamente. En la fase colaborativa, el estudiante aplica y refuerza los conocimientos de la fase conceptual de forma cooperativa durante el tiempo de clase. El docente actúa así como orientador de los estudiantes. En este artículo, se analiza la aplicación de la metodología clase inversa a la sesión práctica 1 de la asignatura Ampliación de Máquinas Eléctricas, del Grado en Ingeniería Eléctrica (Universitat Politècnica de València). En ella, los estudiantes deben analizar seis máquinas eléctricas reales explicadas previamente teóricamente. Los resultados del test calificativo muestran el alto rendimiento académico de la sesión con dicha 
metodología. En concreto, un $75 \%$ de los estudiantes aprobó la prueba y un $23 \%$ de ellos obtuvo calificaciones de sobresaliente. Además, las respuestas de la encuesta verifican la estrecha relación entre el grado de desarrollo de la fase conceptual y el de aprovechamiento de la sesión.

Palabras clave: clase inversa, aprendizaje flexible, aprendizaje autónomo, trabajo colaborativo, estudiantes activos, Taxonomía de Bloom.

\section{Introducción}

El desarrollo de nuevos métodos de trabajo, la introducción de revolucionarias herramientas informáticas junto con la continua investigación en diversos campos de trabajo han provocado profundos cambios en la sociedad en la que vivimos. Uno de los sectores en los que esta evolución ha sido más notoria es la educación (PABLOS and VILLACIERVOS, 2005; De Pablos Pons, 2007; Baelo and Cantón, 2009). En concreto, la educación universitaria está experimentando un proceso dinámico de cambio como resultado al acuerdo del Espacio Europeo de Educación Superior (EEES) y el Proceso de Bolonia (EHEA, 2020). Esta iniciativa se fundamenta en tres pilares: conseguir un sistema universitario europeo común, focalizar la atención en el trabajo de los estudiantes y de los resultados de aprendizaje e incrementar la movilidad y empleabilidad de estudiantes, profesorado y personal de administración y servicios.

Para la consecución de estos objetivos, el sistema de enseñanza superior está sufriendo un proceso dinámico de cambio desde el aprendizaje tradicional hacia un aprendizaje más abierto y flexible (Ginés Mora, 2004; De Miguel Díaz, 2005). En este último, los estudiantes se convierten en sujetos activos dentro de la clase, siendo capaces de detectar sus necesidades de aprendizaje a través de trabajo autónomo y colaborativo con el resto de compañeros. El docente adquiere el papel de orientador del alumnado, que los guía y asesora hacia la consecución de las metas educativas establecidas (Tourón and Santiago, 2015).

Siguiendo esta línea, los profesores de química Jonathan Bergmann y Aaron Sams desarrollaron la metodología de clase inversa o flipped classroom (BERGMANN and SAMS, 2012). Este método se divide en dos fases principales: fase conceptual (fuera del aula) y fase colaborativa (dentro del aula). Durante la fase conceptual, el estudiante de forma individual desarrolla las labores de aplicar, comprender y recordar los conceptos de la clase que se trabajarán posteriormente en el aula. Estas labores corresponden a la base de la Taxonomía de Bloom (ANDERSON et al., 2001). Para ello, dispone de recursos de la materia como apuntes, libros, vídeos, fichas de prácticas...que el docente ha preparado previamente para esta fase. Estos recursos suelen depositarse en aulas virtuales con acceso compartido entre el docente y el estudiante. Durante la fase colaborativa, el estudiante realiza actividades de trabajo cooperativo con el resto de compañeros durante el tiempo de clase que le permiten aplicar y reforzar los conocimientos adquiridos durante la fase conceptual. En el aula se desarrollan así las labores de crear, evaluar y analizar, correspondientes a los niveles superiores de la taxonomía de Bloom, tal y como se muestra en la Figura 1. 


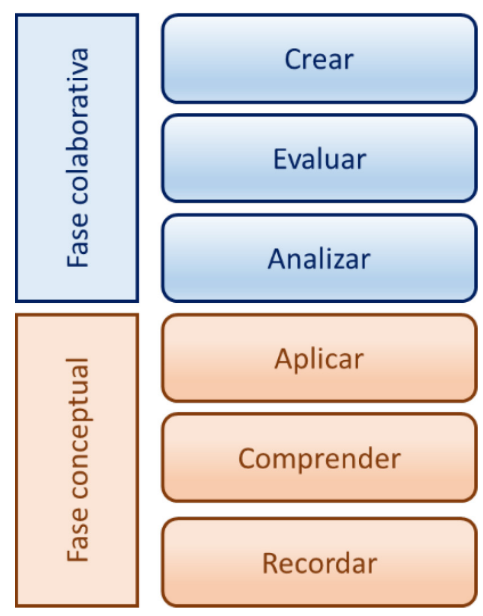

Figura 1. Taxonomía de Bloom

Esta metodología queda alineada con los objetivos de proceso dinámico de cambio en la educación superior. La aplicación de este método en diversos casos de estudio demuestra una mejora del aprendizaje de los estudiantes, aumento de la motivación y desarrollo del trabajo autónomo. También, una mayor participación de los alumnos y mejores resultados académicos (MARTÍNEZ and RUIZ, 2019).

Debido a los positivos resultados obtenidos en anteriores estudios, se ha decidido aplicar la metodología clase inversa en la sesión práctica 1 de la asignatura Ampliación de Máquinas Eléctricas del tercer curso del Grado en Ingeniería Eléctrica de la Universitat Politècnica de València. En esta sesión, los estudiantes analizan seis máquinas eléctricas, estudiadas de forma teórica previamente. Así, pueden aplicar y relacionar los conocimientos teóricos de forma práctica.

Esta comunicación se organiza tal y como se describe a continuación. La sección 1 presenta los objetivos de la comunicación. La sección 2 muestra el desarrollo de la innovación presentada. Los resultados obtenidos se encuentran en el apartado 3. Finalmente, las conclusiones de esta comunicación se desarrollan en el apartado 4.

\section{Objetivos}

El objetivo principal de este trabajo es mejorar el grado de aprovechamiento de la sesión práctica 1 de la asignatura Ampliación de Máquinas Eléctricas mediante la metodología de clase inversa.

De forma paralela al objetivo principal, nos hemos planteado una serie de objetivos específicos:

- Potenciar el trabajo autónomo del estudiante.

- Desarrollar el pensamiento crítico de los alumnos y alumnas (UPV, 2019c)

- Impulsar el trabajo en equipo entre estudiantes (UPV, 2019d)

- Facilitar el aprendizaje del alumnado mediante la aplicación práctica de los conocimientos teóricos y su posterior relación (UPV, 2019a). 


\section{Desarrollo de la innovación}

El modelo pedagógico aula inversa se ha aplicado en la sesión práctica 1 de la asignatura Ampliación de Máquinas Eléctricas (12017). Dicha asignatura se imparte en el tercer curso y semestre A del Grado en Ingeniería Eléctrica de la Universitat Politècnica de València (UPV), (UPV, 2019b). El número de estudiantes totales matriculados en la asignatura durante el curso 2019-2020 ha sido 80. Sin embargo, para poder dedicar una atención más personalizada al alumnado, existen 4 grupos de prácticas con capacidad máxima de 24 estudiantes/grupo. De este modo, la sesión práctica analizada se llevará a cabo con un máximo de 24 alumnos.

\subsection{Dinámica de la clase}

La sesión práctica 1, titulada "Construcción y funcionamiento de las máquinas eléctricas rotatorias", se desarrolla en el laboratorio de máquinas eléctricas del Departamento de Ingeniería Eléctrica UPV. En esta sesión, el alumnado debe analizar seis máquinas eléctricas dinámicas reales distintas, que corresponden con las estudiadas en las sesiones teóricas. De este modo, el estudiante puede aplicar de forma práctica los conocimientos teóricos y relacionarlos, facilitando así su aprendizaje y adquisición de conceptos (CRUJEIRAS and JIMÉNEZ, 2015).

Las distintas fases que componen la sesión práctica analizada se muestran en la Figura 2 y se describen a lo largo de esta sección:

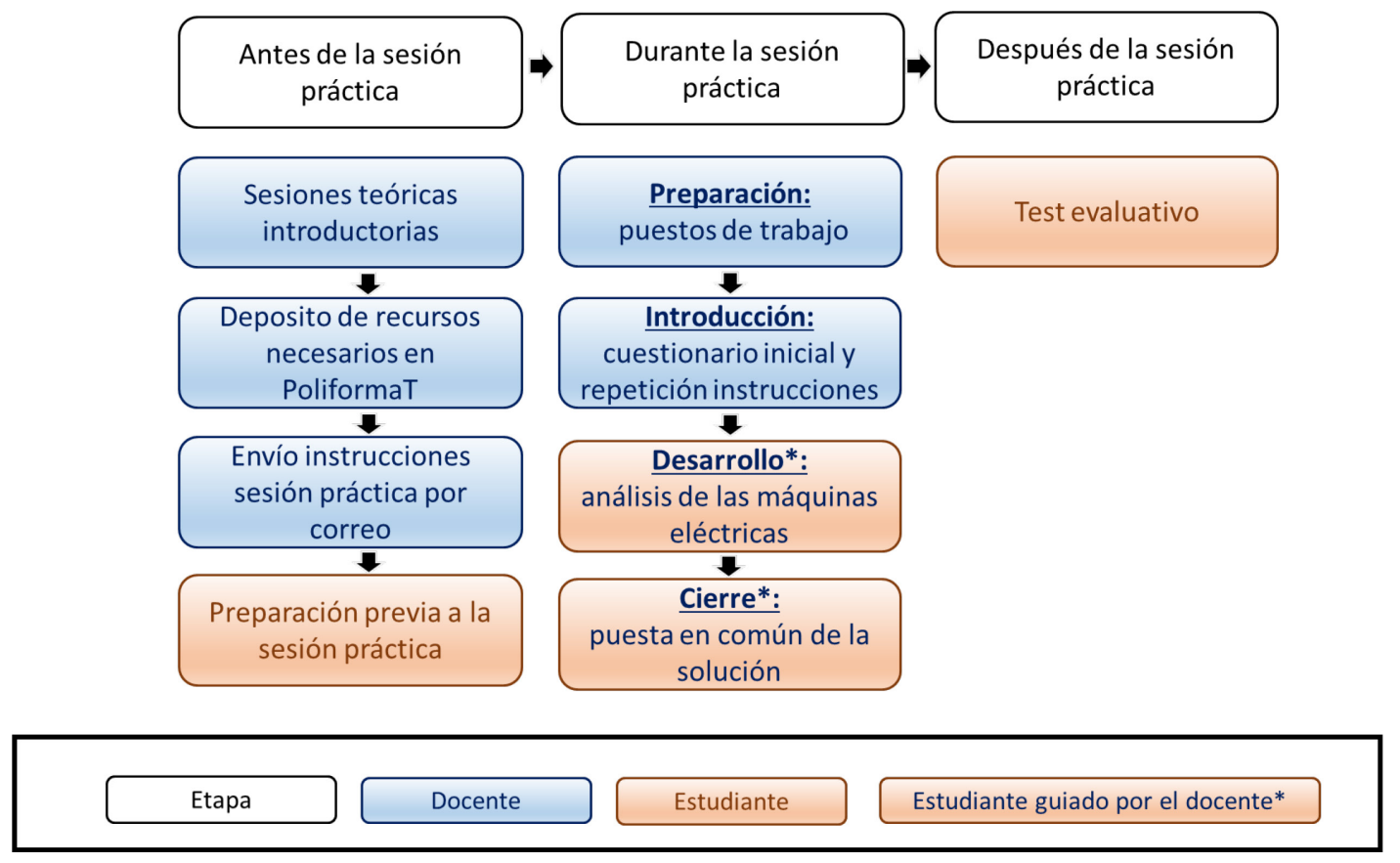

Figura 2. Dinámica de la clase.

\section{Antes de la sesión práctica}

Antes de la primera sesión práctica, los estudiantes reciben las sesiones teóricas iniciales "Introducción a las máquinas eléctricas dinámicas" y "Principios de la conversión mecanoeléctrica", disponibles en los recursos electrónicos PoliformaT. PoliformaT es la plataforma virtual de código abierto utilizada por la 
UPV como herramienta de gestión para la docencia (Mataix et al., 2006), que actúa como repositorio de recursos y facilita la comunicación bidireccional profesorado-alumnado. Así, los estudiantes disponen de los conocimientos básicos iniciales para poder comprender el funcionamiento de las máquinas eléctricas y las principales diferencias entre unos tipos u otros.

Los profesores de prácticas depositan en PoliformaT fichas de cada una de las máquinas a analizar dos semanas antes del inicio de la primera sesión. Además, envían un correo donde notifican la existencia de este recurso e indican la labor que deben llevar a cabo los estudiantes en la sesión. Así, los alumnos disponen de información específica de la tarea a desarrollar en la sesión de laboratorio.

Con la información teórica de la asignatura y la información específica preparada exclusivamente para la sesión práctica, los alumnos están listos para desarrollar la parte conceptual de la sesión práctica en casa (ANDERSON et al., 2001).

\section{$\underline{\text { Sesión práctica }}$}

\section{Preparación}

Antes de iniciar la sesión práctica, los profesores colocan cada máquina eléctrica a analizar en un puesto de trabajo distinto. En total, hay seis máquinas: síncrona brushless, corriente continua con imanes permanentes, reluctancia variable, asíncrona con rotor de jaula de ardilla, flujo axial y síncrona alternador.

\section{$\underline{\text { Introducción }}$}

Durante los 10 primeros minutos de la sesión práctica, el docente vuelve a repetir las instrucciones a los estudiantes. En concreto, les explica que deben formarse un total de 6 subgrupos, con 4 estudiantes máximo por subgrupo. Cada subgrupo deberá analizar en un tiempo aproximado de 15 minutos y en la franja horaria correspondiente la máquina eléctrica mostrada en las instrucciones (Figura 3). Para ello, deben contestar las preguntas mostradas en la ficha (Tabla 1):

\begin{tabular}{|l|c|c|c|c|c|c|}
\cline { 2 - 7 } \multicolumn{1}{c|}{} & $\mathbf{8 : 1 0 - 8 : 2 5}$ & $\mathbf{8 : 2 5} \mathbf{- 8 : 4 0}$ & $\mathbf{8 : 4 0 - 8 : 5 5}$ & $\mathbf{8 : 5 5 - 9 : 1 0}$ & $\mathbf{9 : 1 0 - 9 : 2 5}$ & $\mathbf{9 : 2 5} \mathbf{- 9 : 4 0}$ \\
\hline Subgrupo 1 & $\begin{array}{c}\text { Síncrona } \\
\text { Brushless }\end{array}$ & $\begin{array}{c}\text { CC imanes } \\
\text { permanentes }\end{array}$ & $\begin{array}{c}\text { Reluctancia } \\
\text { variable }\end{array}$ & $\begin{array}{c}\text { Asíncrona } \\
\text { jaula ardilla }\end{array}$ & Flujo axial & $\begin{array}{c}\text { Síncrona } \\
\text { alternador }\end{array}$ \\
\hline Subgrupo 2 & $\begin{array}{c}\text { Síncrona } \\
\text { alternador }\end{array}$ & $\begin{array}{c}\text { Síncrona } \\
\text { Brushless }\end{array}$ & $\begin{array}{c}\text { CC imanes } \\
\text { permanentes }\end{array}$ & $\begin{array}{c}\text { Reluctancia } \\
\text { variable }\end{array}$ & $\begin{array}{c}\text { Asíncrona } \\
\text { jaula ardilla }\end{array}$ & Flujo axial \\
\hline Subgrupo 3 & Flujo axial & $\begin{array}{c}\text { Síncrona } \\
\text { alternador }\end{array}$ & $\begin{array}{c}\text { Síncrona } \\
\text { Brushless }\end{array}$ & $\begin{array}{c}\text { CC imanes } \\
\text { permanentes }\end{array}$ & $\begin{array}{c}\text { Reluctancia } \\
\text { variable }\end{array}$ & $\begin{array}{c}\text { Asíncrona } \\
\text { jaula ardilla }\end{array}$ \\
\hline Subgrupo 4 & $\begin{array}{c}\text { Asíncrona } \\
\text { jaula ardilla }\end{array}$ & Flujo axial & $\begin{array}{c}\text { Síncrona } \\
\text { alternador }\end{array}$ & $\begin{array}{c}\text { Síncrona } \\
\text { Brushless }\end{array}$ & $\begin{array}{c}\text { CC imanes } \\
\text { permanentes }\end{array}$ & $\begin{array}{c}\text { Reluctancia } \\
\text { variable }\end{array}$ \\
\hline Subgrupo 5 & $\begin{array}{c}\text { Reluctancia } \\
\text { variable }\end{array}$ & $\begin{array}{c}\text { Asíncrona } \\
\text { jaula ardilla }\end{array}$ & Flujo axial & $\begin{array}{c}\text { Síncrona } \\
\text { alternador }\end{array}$ & $\begin{array}{c}\text { Síncrona } \\
\text { Brushless }\end{array}$ & $\begin{array}{c}\text { CC imanes } \\
\text { permanentes }\end{array}$ \\
\hline Subgrupo 6 & $\begin{array}{c}\text { CC imanes } \\
\text { permanentes }\end{array}$ & $\begin{array}{c}\text { Reluctancia } \\
\text { variable }\end{array}$ & $\begin{array}{c}\text { Asíncrona } \\
\text { jaula ardilla }\end{array}$ & Flujo axial & $\begin{array}{c}\text { Síncrona } \\
\text { alternador }\end{array}$ & $\begin{array}{c}\text { Síncrona } \\
\text { Brushless }\end{array}$ \\
\hline
\end{tabular}

Figura 3. Instrucciones sesión práctica 1. Ampliación de Máquinas Eléctricas. 
Tabla 1. Ficha sesión práctica 1. Ampliación de Máquinas Eléctricas.

\begin{tabular}{l}
\hline \multicolumn{1}{c}{ Cuestiones } \\
\hline ¿Qué tipo de máquina estás analizando? \\
¿Cuál es el sistema inductor y el sistema inducido? \\
¿Cuántos números de pares de polos tiene la máquina? \\
¿Cuál es su principio de funcionamiento? \\
¿Cuál es su uso más generalizado, motor o generador? \\
\hline
\end{tabular}

En esta fase de la práctica, los docentes realizan también una rápida encuesta a los alumnos para conocer su grado de preparación para la clase inversa.

Tabla 2. Encuesta grado de preparación de los estudiantes para la clase

\begin{tabular}{cc}
\hline \multicolumn{1}{c}{ Cuestión } & Respuestas \\
\hline ¿Has asistido a las sesiones previas teóricas de la asignatura? & $\mathrm{Si} / \mathrm{No}$ \\
¿Has preparado previamente la sesión práctica? & $\mathrm{Si} / \mathrm{No}$ \\
\hline
\end{tabular}

\section{$\underline{\text { Desarrollo }}$}

Durante el desarrollo de la sesión, los alumnos analizan las máquinas eléctricas en cuestión de acuerdo con la organización mostrada en las instrucciones (Figura 3) y contestando las preguntas de la ficha (Tabla 1). Esta fase tiene una duración total de 1 hora y media (15 minutos/máquina). Los estudiantes trabajan de forma cooperativa en equipo y autónoma respecto el docente (MARTÍNEZ and RUIZ, 2019), donde este último simplemente les asiste en momentos puntuales resolviendo las cuestiones planteadas. Así, en el tiempo de clase se desarrollan los niveles superiores de la taxonomía de Bloom (ANDERSON et al., 2001) ya que el desarrollo conceptual se llevó a cabo previamente antes de la sesión práctica.

\section{$\underline{\text { Cierre }}$}

Durante los últimos 20 minutos, los distintos subgrupos ponen en común el análisis realizado para cada máquina eléctrica hasta llegar a la solución del problema. En esta etapa, el profesor guía también a los alumnos.

\section{Después de la sesión práctica}

Al inicio de la siguiente sesión práctica (sesión práctica 2), los estudiantes realizan la prueba de evaluación correspondiente a la sesión práctica 1. Esta prueba de evaluación consiste en un test PoliformaT de 5 preguntas relacionadas con al análisis realizado en la sesión práctica 1 , con 4 posibles respuestas, a realizar en 10 minutos. 


\subsection{Valoración}

Tal y como muestran diversos estudios (Harden and Crosby, 2000), la introducción de nuevas metodologías educacionales distintas de las tradicionales requiere de un seguimiento y evaluación. En este caso de estudio, se valoró la efíciencia de la clase inversa en la sesión práctica 1 a través de tres puntos de control:

- $\quad$ Calificaciones del test PoliformaT. Con estas calificaciones se ha podido observar el grado de aprovechamiento genérico de los estudiantes al utilizar la metodología clase inversa. Se han comparado además con los resultados obtenidos por un grupo de estudiantes que no realizó la experiencia clase inversa, para comprobar así la relevancia de dicha metodología en la sesión práctica analizada.

- Respuestas de la encuesta sobre el grado de preparación para la clase inversa. Con dicha encuesta (Tabla 2) se ha podido analizar el estado de preparación de los estudiantes para la clase inversa y, por tanto, el desarrollo de la fase conceptual.

- Comparativa entre calificaciones del test PoliformaT y respuestas de la encuesta sobre el grado de preparación para la clase inversa. Con esta comparativa se ha podido analizar el grado de aprovechamiento específico de la metodología clase inversa en función de la preparación previa por parte del estudiante.

\section{Resultados}

En este apartado se presentan los resultados de valoración de la metodología clase inversa en la sesión práctica 1 de Máquinas Eléctricas. Por un lado, se muestran las calificaciones del test PoliformaT utilizado como sistema de evaluación de dicha práctica y las respuestas de la encuesta sobre el grado de preparación para la clase. Por otro lado, se muestra el cruce de información entre ambos resultados.

\subsection{Calificaciones Test PoliformaT}

La Figura 4 refleja las calificaciones obtenidas por los estudiantes en el test PoliformaT evaluativo de la sesión práctica 1 . El rango de aprobados (nota $\geq 5$ ) ha sido de un $75 \%$, frente a un $25 \%$ de suspendidos (nota $<5$ ), tal y como muestra la Figura 4.a. Analizando los estudiantes suspendidos (Figura 4.b), el mayor porcentaje (10\%) obtuvo notas cercanas al aprobado, entre 3.5 y 5 . Por otro lado, la mayor parte de los aprobados obtuvo calificaciones entre 5 y 7 y entre 7 y 9 , respectivamente (26\%). Finalmente, un porcentaje también muy alto de estudiantes $(23 \%)$ alcanzó notas sobresalientes, con calificaciones superiores a 9. De forma genérica, todos estos resultados muestran cuantitativamente el alto grado de aprovechamiento y positivo impacto de la clase inversa en los estudiantes. 


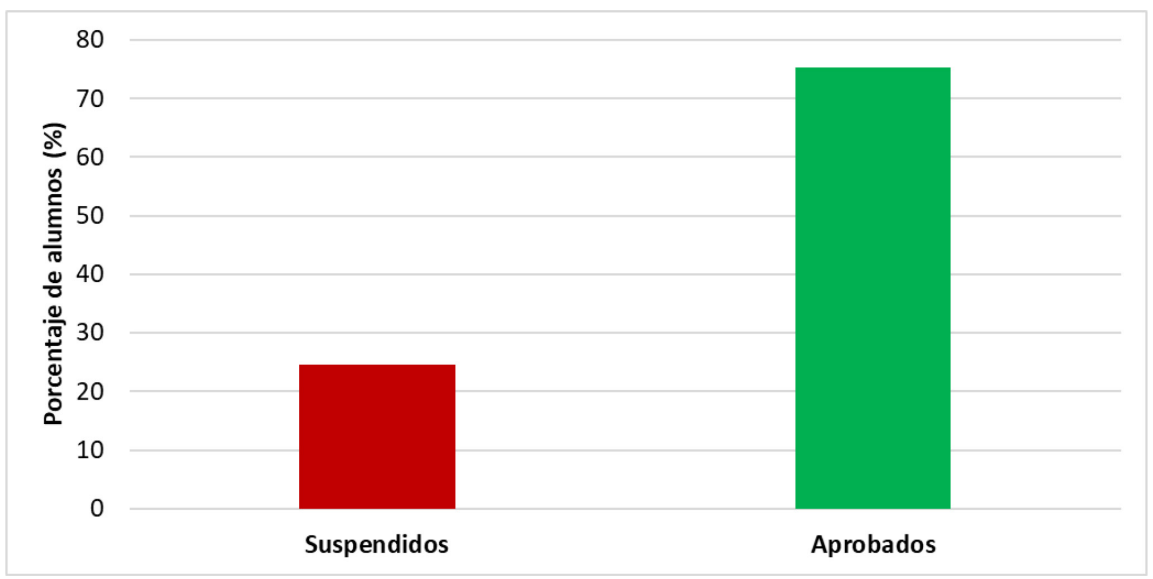

(a)

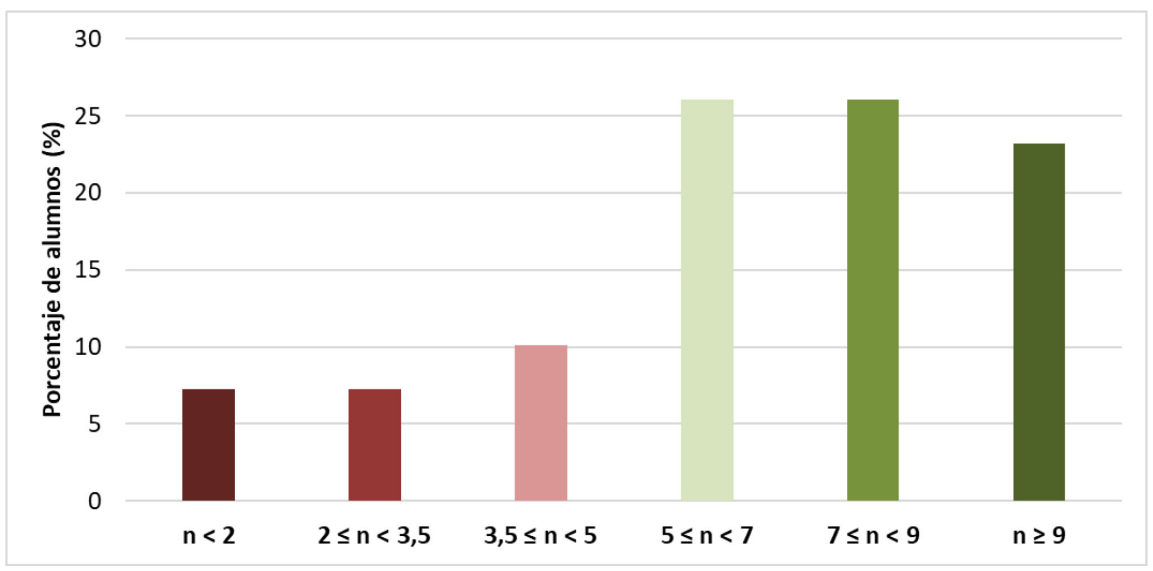

(b)

Figura 4. Calificaciones test PoliformaT sesión práctica 1. (a) Suspendidos/aprobados. (b) Rangos.

Para poder evaluar la relevancia de esta metodología, los resultados obtenidos en la realización del test PoliformaT se han comparado con los obtenidos por un grupo de estudiantes que no realizó la experiencia clase inversa. La Figura 5 refleja esta comparativa. En ella, se puede ver como el rango de aprobados usando la metodología tradicional fue de un $55 \%$, frente a un $75 \%$ usando la clase inversa. Además, las franjas de calificaciones usando la clase tradicional fueron también más bajas comparadas con la clase inversa. El mayor rango de calificación obtenido mediante clase tradicional fue de un 35\% y corresponde al aprobado (notas entre 5 y 7), seguido del suspenso alto (notas entre 3.5 y 5 ) que representaba un 20\%. Sin embargo, en la clase inversa las franjas de aprobado y suspenso alto tenían una representación del $26 \%$ y $10 \%$ respectivamente. Además, con la metodología tradicional sólo un $5 \%$ de los estudiantes alcanzó notas de sobresaliente, frente a un $23 \%$ cuando la metodología fue clase inversa. 
Con esta comparativa se comprueba la idoneidad de la metodología clase inversa frente a la clase tradicional en la sesión práctica analizada en esta comunicación.

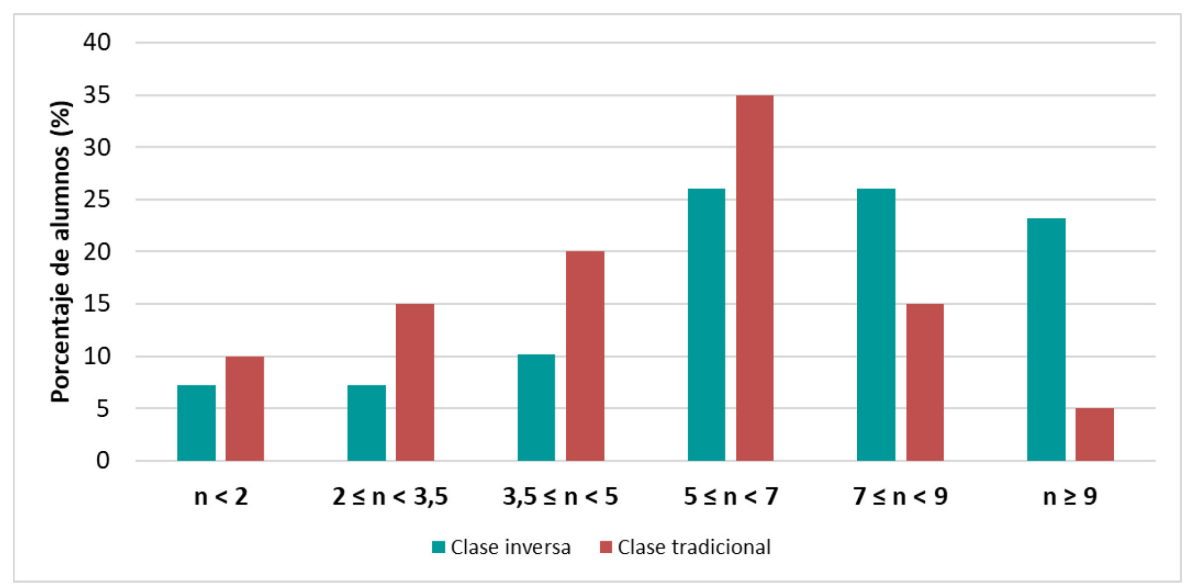

Figura 5. Comparativa calificaciones test Poliformat clase inversa-clase tradicional.

\subsection{Respuestas de la encuesta sobre el grado de preparación para la clase inversa}

La Figura 6 muestra los resultados de la encuesta inicial realizada por los estudiantes en la sesión práctica respecto a su grado de preparación para la clase inversa. Se observa que la mayor parte de los estudiantes asistió a las clases previas de teoría, un $84 \%$. Un porcentaje menor, aunque considerable, preparó también inicialmente la práctica $(71 \%)$. De forma total, un $71 \%$ de los estudiantes llevó a cabo de forma completa la fase de preparación inicial de la clase inversa (asistencia clases de teoría y preparación inicial práctica), lo cual se considera un porcentaje alto.

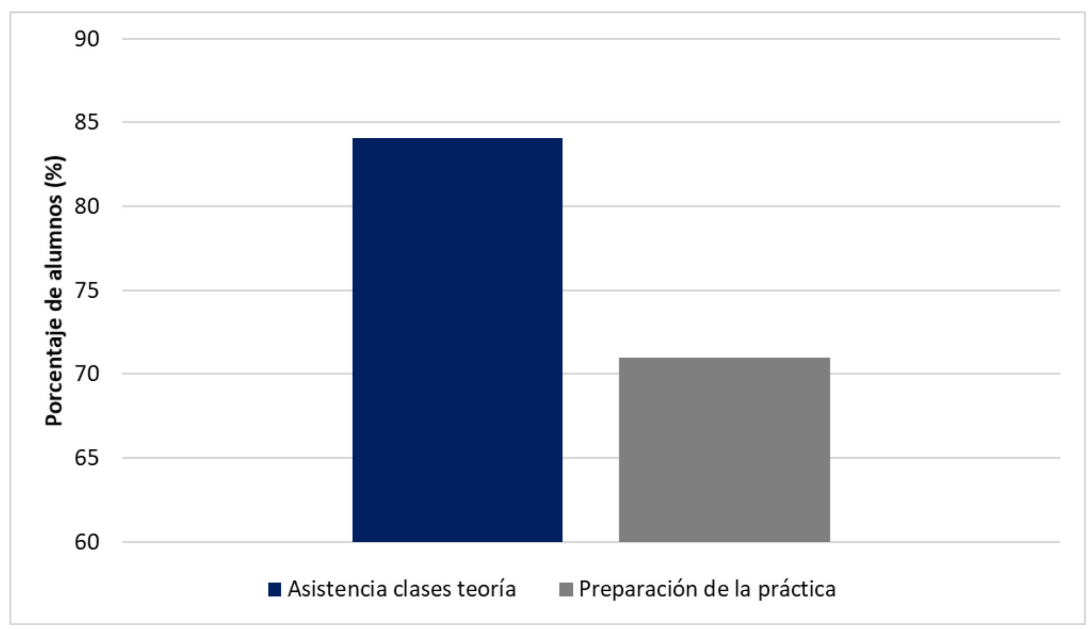

Figura 6. Respuestas de la encuesta sobre el grado de preparación para la clase inversa. 


\subsection{Comparativa entre calificaciones del test PoliformaT y respuestas de la encuesta sobre el grado de preparación para la clase inversa}

Finalmente, se ha creído conveniente realizar un cruce de datos entre las calificaciones del test PoliformaT y las respuestas obtenidas en la encuesta sobre el grado de preparación de los estudiantes para la clase inversa. Los resultados quedan reflejados en la Figura 7.

De forma genérica, se comprueba que los estudiantes que llevaron a cabo el desarrollo completo de la fase conceptual (asistieron a las clases teóricas y prepararon previamente la práctica) son los que mejor aprovechamiento tuvieron de la clase inversa, obteniendo así las mejores calificaciones en el test. En concreto, el 100\% del alumnado que obtuvo calificaciones de sobresaliente asistió a las clases teóricas y preparó previamente la sesión práctica. Por el contrario, de los estudiantes con peores calificaciones (nota $<2$ ), tan sólo el 20\% había asistido a las clases de teoría y ninguno de ellos había preparado la práctica.

Queda así reflejada la eficacia de la metodología clase inversa en la sesión práctica analizada, así como la necesidad de llevar a cabo el desarrollo de la fase conceptual previo para poder aprovechar este tipo de metodología al máximo.

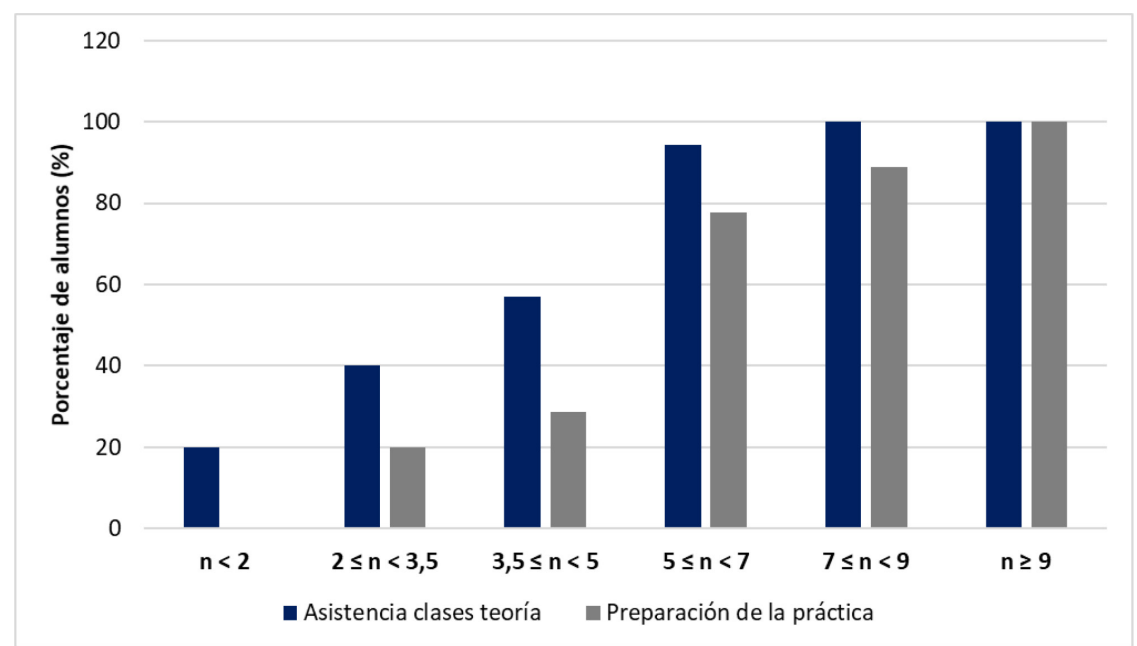

Figura 7. Comparativa entre calificaciones del test PoliformaT y grado de preparación para la clase inversa. 


\section{Conclusiones}

La educación superior se encuentra sumergida en un proceso de transformación dinámica, basada en el acuerdo del EEES y el Proceso Bolonia. La nueva enseñanza, a diferencia de la tradicional, centra la atención en los estudiantes, que desarrollan un trabajo autónomo y colaborativo de aprendizaje, mientras que el docente actúa como orientador de los mismos. Surge así la metodología clase inversa, basada en una primera fase conceptual individual del estudiante fuera del aula y una fase de trabajo colaborativo con el resto de compañeros guiado por el profesor dentro del aula.

Esta comunicación describe la aplicación de la metodología clase inversa en la primera sesión práctica de la asignatura Ampliación de Máquinas Eléctricas del Grado en Ingeniería Eléctrica de la UPV. En esta sesión, los estudiantes desarrollan la fase conceptual de forma individual previamente a través de los recursos preparados por los profesores de prácticas y las clases teóricas previas recibidas. Durante la sesión práctica, los estudiantes analizan las máquinas eléctricas dispuestas en el laboratorio por equipos y de forma autónoma. El docente les asiste y resuelve sus dudas en todo momento. En la última parte de la práctica, los estudiantes ponen en común los resultados alcanzados y reciben el feedback del docente.

La introducción de un cambio metodológico como el aquí descrito requiere de un proceso de seguimiento y evaluación para comprobar su eficiencia. En concreto, para la sesión en cuestión se valoró el grado de aprovechamiento de la metodología clase inversa a través de las calificaciones numéricas obtenidas en el test evaluativo PoliformaT de dicha sesión, las respuestas a la encuesta sobre el grado de preparación de los estudiantes para la clase inversa y finalmente a través de una comparativa de los dos puntos anteriores.

En primer lugar, las calificaciones numéricas del test PoliformaT fueron bastante altas, lo cual muestra el alto grado de aprovechamiento y positivo impacto de la clase inversa. Un 75\% de los estudiantes aprobó el test. Además, una gran parte del alumnado obtuvo calificaciones entre 5 y 7 y entre 7 y 9 (26\% y $26 \%$, respectivamente) y hasta un $23 \%$ alcanzó calificaciones de sobresaliente. Paralelamente, se compararon las calificaciones de dicho test con las obtenidas por un grupo de estudiantes que no realizó la metodología clase inversa. El rango de estudiantes aprobados con la metodología tradicional descendió hasta el 55\% y las calificaciones descendieron también de forma general: el $20 \%$ y $35 \%$ de los estudiantes obtuvo calificaciones entre 3.5 y 5 , y entre 5 y 7 respectivamente, y sólo un $5 \%$ consiguió calificaciones de sobresaliente. Esta comparativa demuestra idoneidad de la metodología clase inversa en la sesión práctica analizada.

Respecto al grado de preparación para la clase inversa, la encuesta en cuestión muestra que hasta un $71 \%$ del alumnado realizó por completo las dos tareas preparatorias para la misma. Se considera este porcentaje bastante alto.

Por último, la comparativa entre las calificaciones numéricas del test PoliformaT y los resultados de la encuesta reflejan el alto grado de relación entre el desarrollo de la fase conceptual y el grado de aprovechamiento de la clase inversa. En concreto, el 100\% de los estudiantes con las calificaciones más altas (>9) habían realizado por completo las dos tareas de preparación. Sin embargo, sólo el $20 \%$ y el $0 \%$ de los estudiantes con las calificaciones más bajas $(<2)$ había asistido a las clases teóricas y había preparado la práctica respectivamente.

Con todos los resultados recopilados, se demuestra la eficacia de la metodología clase inversa en su aplicación al caso de estudio. Como trabajo futuro, se quiere conseguir que un mayor porcentaje de estudiantes desarrolle por completo la fase previa a la clase inversa para aumentar así el grado de aprovechamiento de la misma. 


\section{Agradecimientos}

Este trabajo ha sido respaldado en parte por la administración pública de la Generalitat Valencia bajo la beca ACIF/2018/106.

\section{Referencias}

ANDERSON, L. W. et al. (2001) A Taxonomy for Learning, Teaching and Assessing. A Revision of Bloom's Taxonomy of Educational Objectives. Edited by Pearson.

BAELO, R. AND CANTÓN, I. (2009) 'Las tecnologías de la información y la comunicación en la educación superior. Estudio descriptivo y de revisión', Revista Iberoamericana de Educación, 50(7), pp. $1-12$.

BERGMANN, J. and SAMS, A. (2012) Flip Your Classroom: Reach Every Student in Every Class Every Day. Edited by INTL SOCIETY FOR TECHNOLOGY ED.

CRUJEIRAS, B. and JIMÉNEZ, M. P. (2015) 'Desafíos planteados por las actividades abiertas de indagación en el laboratorio: articulación de conocimientos teóricos y prácticos en las prácticas científicas', Enseñanza de las ciencias: revista de investigación y experiencias didácticas, pp. 63-84.

EHEA (2020) European Higher Education Area and Bologna Process. Available at: http://www.ehea.info/ (Accessed: 27 February 2020).

GINÉS MORA, J. (2004) 'La necesidad del cambio educativo para la sociedad del conocimiento', Revista Iberoamericana de la Educación, 35, pp. 13-37. Available at: https://dialnet.unirioja.es/servlet/articulo?codigo $=1071068$.

HARDEN, R. AND CROSBY, J. (2000) 'The good teacher is more than a lecturer - the twelve roles of the teacher', Medical Teacher, 22, pp. 334-347.

MARTÍNEZ, R. and RUIZ, M. C. (2019) 'Estudiantes motivados y participativos. ¿Una utopía? No, una realidad gracias al aula invertida', IN-RED 2019: V Congreso de Innovación Educativa y Docencia en Red. Edited by Editorial Universitat Politècnica de Valencia. Valencia, pp. 364-377.

MATAIX, J. B. ET AL. (2006) PoliformaT: una estrategia para la formación on-line en la Educación Superior. Available at: http://www.virtualeduca.org (Accessed: 16 July 2020).

DE MIGUEL DÍAZ, M. (2005) 'Cambio de paradigma metodológico en la Educación Superior Exigencias que conlleva', Cuadernos de Integración Europea, pp. 16-27.

PABLOS, J. and VILLACIERVOS, P. (2005) 'El espacio Europeo de Educación Superior y las tecnologías de la información y la comunicación. Percepciones y demandas del profesorado', Revista de Educación, 337, pp. 99-124.

De Pablos Pons, J. (2007) 'El cambio metodológico en el Espacio Europeo de Educación Superior y el papel de las tecnologías de la información y la comunicación', RIED: Revista iberoamericana de educación a distancia, 10 (2), pp. 15-44.

TOURÓN, J. AND SANTIAGO, R. (2015) 'El modelo Flipped Learning y el desarrollo del talento en la escuela', Revista de Educacion, (368), pp. 33-65. doi: 10.4438/1988-592X-RE-2015-368-288.

UPV (2019a) Aplicación y pensamiento práctico: Competencias Transversales: UPV. Available at: https://www.upv.es/contenidos/COMPTRAN/info/954715normalc.html (Accessed: 26 February 2020).

UPV (2019b) Guía docente Ampliación Máquinas Eléctricas. Available at: https://sede.upv.es/eVerificador (Accessed: 26 February 2020).

UPV (2019c) Pensamiento crítico: Competencias Transversales: UPV. Available at: http://www.upv.es/contenidos/COMPTRAN/info/955136normalc.html (Accessed: 14 March 2019).

UPV (2019d) Trabajo en equipo: Competencias Transversales: UPV. Available at: http://www.upv.es/contenidos/COMPTRAN/info/954872normalc.html (Accessed: 14 March 2019).

(c)) BY-NC-ND 2020, Universitat Politècnica de València

Congreso In-Red (2020) 